ALCOHOLIC LIVER DISEASE

\title{
Analysis of factors predictive of mortality in alcoholic hepatitis and derivation and validation of the Glasgow alcoholic hepatitis score
}

\author{
E H Forrest, C D J Evans, S Stewart, M Phillips, Y H Oo, N C McAvoy, N C Fisher, S Singhal, \\ A Brind, G Haydon, J O'Grady, C P Day, P C Hayes, L S Murray, A J Morris
}

Gut 2005;54:1174-1179. doi: 10.1136/gut.2004.050781

\begin{abstract}
See end of article for authors' affiliations

Correspondence to Dr E H Forrest, Department of Gastroenterology, Glasgow Royal Infirmary, Castle St, Glasgow G4 OSF, UK; Ewan.Forresł@ northglasgow.scot.nhs.uk

Revised version received 23 December 2004

Accepted for publication

11 January 2005
\end{abstract}

\begin{abstract}
Introduction: Alcoholic hepatitis is associated with a high short term mortality. We aimed to identify those factors associated with mortality and define a simple score which would predict outcome in our population.

Methods: We identified 241 patients with alcoholic hepatitis. Clinical and laboratory data were recorded on the day of admission (day 1) and on days 6-9. Stepwise logistic regression was used to identify variables related to outcome at 28 days and 84 days after admission. These variables were included in the Glasgow alcoholic hepatitis score (GAHS) and its ability to predict outcome assessed. The GAHS was validated in a separate dataset of 195 patients.

Results: The GAHS was derived from five variables independently associated with outcome: age $(p=0.001)$ and, from day 1 results, serum bilirubin $(p<0.001)$, blood urea $(p=0.019)$ and, from day $6-9$ results, serum bilirubin $(p<0.001)$, prothrombin time $(p=0.002)$, and peripheral blood white blood cell count $(p=0.001)$. The GAHS on day 1 had an overall accuracy of $81 \%$ when predicting 28 day outcome. In contrast, the modified discriminant function had an overall accuracy of $49 \%$. Similar results were found using information at 6-9 days and when predicting 84 day outcome. The accuracy of the GAHS was confirmed by the validation study of 195 patients The GAHS was equally accurate irrespective of the use of the international normalised ratio or prothrombin time ratio, or if the diagnosis of alcoholic hepatitis was biopsy proven or on the basis of clinical assessment.

Conclusions: Using variables associated with mortality we have derived and validated an accurate scoring system to assess outcome in alcoholic hepatitis. This score was able to identify patients at greatest risk of death throughout their admission.
\end{abstract}

A lcoholic hepatitis is perhaps the most florid manifestation of alcoholic liver disease. The more severe forms of this condition are associated with a high mortality. Identification of those patients at greatest risk of death is vital not only for decisions relating to individual management but also for the design of clinical studies assessing possible treatments for this condition.

In 1978 the discriminant function (DF) was first described in a placebo controlled study of the benefit of corticosteroid therapy in 55 patients with alcoholic hepatitis. ${ }^{1}$ DF was calculated between seven and 12 days after admission. Patients with a DF $>93$ and treated with placebo had a $25 \% 28$ day survival while those with a score $\leqslant 93$ had a survival of 100\%. In 1989 the DF was modified in the context of a further placebo controlled corticosteroid trial involving 66 patients. ${ }^{2}$ A modified DF (mDF) of $>32$ and/or the presence of encephalopathy in placebo treated patients was associated with a $65 \% 28$ day survival. A recent reanalysis of a previously published placebo controlled corticosteroid trial confirmed this observation, with a $68 \% 28$ day survival in placebo treated patients with an $\mathrm{mDF} \geqslant 32$ while those with a score $<32$ had a survival of $93 \%{ }^{3}$ The American College of Gastroenterology has recommended that the mDF be used to assess the severity of alcoholic hepatitis and a threshold of 32 be used to consider corticosteroid therapy. ${ }^{4}$ More recently, the MELD score has been applied to alcoholic hepatitis. In a study of 34 patients identified with a MELD score of $>11$, there was $45 \% 30$ day survival while those with a score $\leqslant 11$ had a survival of $96 \%$ (table 1$)^{5}$
Our own clinical experience indicated that the mDF did not clearly identify those patients at greatest risk and indeed the published studies suggest that most patients with an mDF $>32$ survive without treatment. We wished to identify those factors associated with a poor outcome in our population of patients with alcoholic hepatitis. The ultimate aim was to create and validate a simple clinically useful score which was easily calculable from readily available variables, and which may better inform decisions regarding treatment by more accurately identifying patients with higher mortality than the currently available scoring systems.

\section{METHODS}

Analysis of prognostic factors and development of the Glasgow alcoholic hepatitis score

We identified patients presenting with alcoholic liver disease to the Glasgow Royal Infirmary and the Victoria Infirmary, Glasgow. Patients had a history of excessive alcohol ingestion until at least three weeks before admission and a serum bilirubin level of $\geqslant 80 \mu \mathrm{mol} / \mathrm{l}$ on admission. No patient was treated with corticosteroids or pentoxifylline. Patients were excluded if they had an inpatient stay of less than 48 hours either because of early death or discharge from hospital, or if they presented with gastrointestinal haemorrhage. Patients with evidence, either initially or subsequently, of viral

Abbreviations: GAHS, Glasgow alcoholic hepatitis score; DF, discriminant function; $\mathrm{mDF}$, modified DF; INR, international normalised ratio; AST, aspartate aminotransferase; ALT, alanine aminotransferase 
Table 1 Scoring systems used in the assessment of alcoholic hepatitis

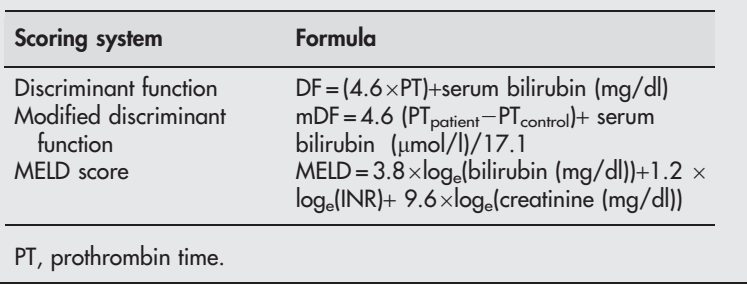

hepatitis, autoimmune liver disease, biliary obstruction, or hepatocellular carcinoma were excluded.

Patient records were reviewed and the findings on clinical examination at the time of admission were documented. Results of standard laboratory tests were also recorded on the day of admission (day 1) and between days 6-9 after admission. The outcome of the admission episode, and short term ( 28 day) and medium term ( 84 day) mortality were also documented.

Stepwise logistic regression was used to identify variables which were independently associated with short and medium term outcome. Based on the variables identified by the logistic regression analysis, a simple scoring system for severity of disease was derived. Comparison of accuracies was performed using $\chi^{2}$ analysis. Analysis was performed using SPSS version 10.0.7.

\section{Validation of the Glasgow alcoholic hepatitis score (GAHS)}

In order to validate the Glasgow alcoholic hepatitis score, we identified patients presenting to eight hospitals throughout the UK: Glasgow, Newcastle, London, Birmingham, Sandwell, Dudley, Edinburgh, and North Staffordshire. All patients had a serum bilirubin level $\geqslant 80 \mu \mathrm{mol} / \mathrm{l}$ and a history of recent alcohol excess. As in the initial dataset, patients with viral hepatitis, autoimmune liver disease, or hepatocellular carcinoma were excluded.

The GAHS was calculated for each patient. mDF was calculated if prothrombin time was measured. The MELD score was calculated if the international normalised ratio (INR) was measured. These scores were calculated on the day of admission (day 1) and on day 7. Outcome was related to survival at day 28 and day 84 . There was inevitably a degree of heterogeneity in recruitment of patients between the different hospitals, as detailed in table 2. No patients was treated with corticosteroids or pentoxifylline. Patients treated with antioxidant therapy were included as no improvement in survival was seen with their use.
Table 3 Study population; 241 unselected patients presenting with a clinical diagnosis of alcoholic hepatitis

\begin{tabular}{lll}
\hline & Day 1 & Days 6-9 \\
\hline Bilirubin $(\mu \mathrm{mol} / \mathrm{l})$ & $155(80-806)$ & $143(18-910)$ \\
PT $(\mathrm{s})$ & $21(14-46)$ & $22(14-124)$ \\
DF & $41(4-166)$ & $45(0-535)$ \\
DF $\geqslant 32(\%)$ & 68 & 67 \\
Encephalopathy (\%) & 30 & - \\
Ascites (\%) & 63 & - \\
\hline
\end{tabular}

Values are median (range) or percentage.

PT, prothrombin time; DF, discriminant function.

Table 4 The Glasgow alcoholic hepatitis score

\begin{tabular}{llll}
\hline & \multicolumn{2}{l}{ Score given } \\
\cline { 2 - 4 } & $\mathbf{1}$ & $\mathbf{2}$ & $\mathbf{3}$ \\
\hline Age $\left(10^{9} / \mathrm{l}\right)$ & $<50$ & $\geqslant 50$ & - \\
WCC & $<15$ & $\geqslant 15$ & - \\
Urea $(\mathrm{mmol} / \mathrm{I})$ & $<5$ & $\geqslant 5$ & - \\
PT ratio & $<1.5$ & $1.5-2.0$ & $>2.0$ \\
Bilirubin $(\mu \mathrm{mol} / \mathrm{l})$ & $<125$ & $125-250$ & $>250$ \\
\hline
\end{tabular}

PT, prothrombin time; WCC, white cell count.

\section{RESULTS}

\section{Analysis of prognostic factors and derivation of the GAHS}

Patient population

In total, 241 patients were identified. Their clinical characteristics are shown in table 3. Overall survival was $77 \%$ and $68 \%$ at days 28 and 84, respectively. Patients with an admission $\mathrm{mDF}$ of $\geqslant 32$ had a survival of $71 \%$ and $62 \%$ at days 28 and 84, respectively.

\section{Logistic regression}

Variables considered predictive of 28 and 84 day outcome were age, sex, the presence of ascites, the presence of encephalopathy, day l sodium, day l blood urea, and serum bilirubin, serum albumin, alanine aminotransferase (ALT), aspartate aminotransferase (AST), the ALT to AST ratio, alkaline phosphatase, prothrombin time, and peripheral white blood cell count (all on day 1 and on days 6-9).

Factors independently associated with 28 day mortality identified by stepwise logistic regression were age $(p=0.001)$ and, from day 1 results, serum bilirubin $(p<0.001)$, serum albumin $(p=0.025)$, blood urea $(p=0.019)$, and the presence of encephalopathy $(\mathrm{p}=0.028)$ and, from day $6-9$ results, serum bilirubin $(\mathrm{p}<0.001)$, prothrombin time

Table 2 Selection of patients for validation of the Glasgow alcoholic hepatitis score

\begin{tabular}{|c|c|c|}
\hline Hospital & No of Patients & Inclusion criteria \\
\hline Glasgow & 15 & GI bleeders excluded \\
\hline Birmingham & 24 & Gl bleeders excluded \\
\hline Newcastle & 31 & Part of an RCT comparing antioxidants with placebo; $\mathrm{mDF} \geqslant 32$ \\
\hline London & 46 & $\begin{array}{l}\text { Part of an RCT comparing antioxidants with corticosteroids. } \mathrm{mDF} \geqslant 32 \text {, } \\
\text { serum bilirubin }>100 \mu \mathrm{mol} / \mathrm{I} \text {, serum creatinine }<500 \mu \mathrm{mol} / / \mathrm{I} \text {. No } \\
\text { active sepsis or } \mathrm{Gl} \text { bleeding for } 48 \text { hours }\end{array}$ \\
\hline North Staffordshire & 28 & GI bleeders excluded \\
\hline Dudley/Sandwell & 37 & Patients with recent $\mathrm{Gl}$ bleeding included \\
\hline Edinburgh & 14 & GI bleeders excluded \\
\hline
\end{tabular}

All patients had a serum bilirubin level $\geqslant 80 \mu \mathrm{mol} / \mathrm{l}$, a history of recent alcohol excess, and an aspartate aminotransferase level of $<500 \mathrm{IU} / \mathrm{l}$.

$\mathrm{Gl}$, gastrointestinal; mDF, modified discriminant function; RCT, randomised controlled trial. 


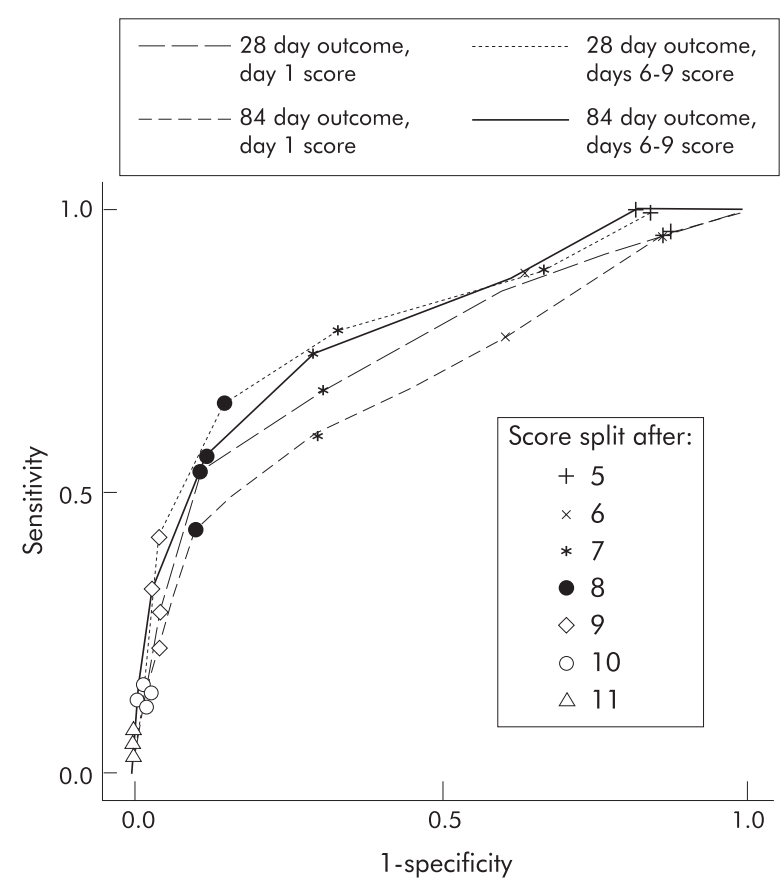

Figure 1 Receiver operating curve of the Glasgow alcoholic hepatitis score at day 1 and days 6-9, used to predict outcome at 28 and 84 days.

$(\mathrm{p}=0.002)$, and peripheral blood white blood cell count $(\mathrm{p}=0.001)$.

Factors independently associated with 84 day mortality identified by stepwise logistic regression were age $(p=0.006)$ and, from day 1 results, serum bilirubin $(p=0.001)$, serum albumin $(p=0.022)$, blood urea $(p=0.001)$, and the AST to ALT ratio $(p=0.018)$ and, from day 6-9 results, serum bilirubin $(p<0.001)$, prothrombin time $(p, 0.001)$, and peripheral blood white blood cell count $(\mathrm{p}=0.009)$.

\section{Development of the scoring system}

Of the variables associated with outcome, we selected five for the derivation of a scoring system: patient age, blood urea $(\mathrm{mmol} / \mathrm{l})$, peripheral blood white cell count $\left(10^{9} / 1\right)$, serum bilirubin $(\mu \mathrm{mol} / \mathrm{l})$, and prothrombin time, expressed as a ratio of the control value. The GAHS was calculated as the sum of the scores derived (table 4). Values obtained ranged from 5 to 12. There was a marked rise in mortality when a score greater than 8 was attained, and a receiver operating curve (fig 1) suggests that using scores greater than 8 to define poor prognosis is optimum for simultaneous maximisation of sensitivity and specificity.

Based on this cut point, the GAHS lacked sensitivity but had a far superior specificity and overall accuracy relative to
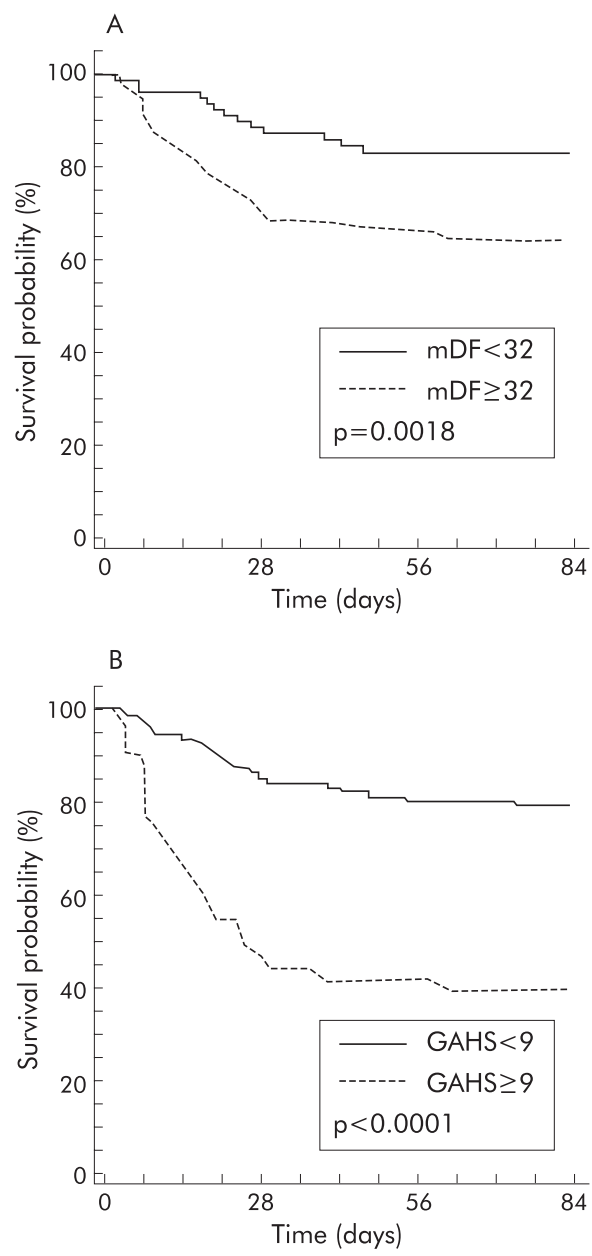

Figure 2 Kaplan-Meier survival analysis relative to the modified discriminant function (mDF) (A) and the Glasgow alcoholic hepatitis score (GAHS) (B).

the mDF (table 5). Kaplan-Meier survival analysis for both $\mathrm{mDF}$ and the GAHS is shown in fig 2.

\section{Validation of the GAHS \\ Patient population}

In total, 195 patients were studied. Of these, 65 (33.3\%) had biopsy proven alcoholic hepatitis. Overall survival was $71 \%$ and $63 \%$ at days 28 and 84, respectively. Patients with an admission $\mathrm{mDF}$ of $\geqslant 32(\mathrm{n}=118)$ had a survival of $64 \%$ and $52 \%$ at days 28 and 84 , respectively. Survival in relation to individual GAHS values as well as for patients with a GAHS value $<9$ or $\geqslant 9$ is shown in table 6 .

Table 5 Sensitivities (Sen), specificities (Spec), positive predictive values (PPV), negative predictive values (NPV), and overall accuracies (Acc) of the Glasgow alcoholic hepatitis score (GAHS) relative to the modified discriminant function (mDF) (derivation dataset)

\begin{tabular}{cll}
\hline & $\begin{array}{l}\text { Day 28 outcome }(\%) \\
\text { (Sen/Spec; PPV/NPV; Acc) }\end{array}$ & $\begin{array}{l}\text { Day 84 outcome }(\%) \\
\text { (Sen/Spec; PPV/NPV; Acc) }\end{array}$ \\
\hline Day 1 data & & \\
GAHS $(</ \geqslant 9)$ & $54 / 89 ; 61 / 86 ; 81$ & $43 / 90 ; 67 / 77 ; 75$ \\
mDF $(</ \geqslant 32)$ & $82 / 39 ; 29 / 88 ; 49$ & $79 / 40 ; 38 / 80 ; 53$ \\
Day 6-9 data & & \\
GAHS $(</ \geqslant 9)$ & $66 / 85 ; 54 / 91 ; 81$ & $56 / 88 ; 67 / 83 ; 78$ \\
mDF $(</ \geqslant 32)$ & $92 / 41 ; 30 / 95 ; 52$ & $88 / 44 ; 41 / 89 ; 57$ \\
\hline
\end{tabular}


Table 6 Survival from alcoholic hepatitis with respect to individual Glasgow alcoholic hepatitis score (GAHS) values (derivation and validation datasets combined)

\begin{tabular}{|c|c|c|}
\hline & $\begin{array}{l}\text { Day } 28 \text { survival } \\
\text { (\%) }\end{array}$ & $\begin{array}{l}\text { Day } 84 \text { survival } \\
\text { (\%) }\end{array}$ \\
\hline \multicolumn{3}{|l|}{ Day 1 score } \\
\hline GAHS 5 & 93 & 86 \\
\hline GAHS 6 & 90 & 79 \\
\hline GAHS 7 & 87 & 81 \\
\hline GAHS 8 & 82 & 73 \\
\hline GAHS 9 & 63 & 56 \\
\hline GAHS 10 & 33 & 31 \\
\hline GAHS 11 & 31 & 19 \\
\hline GAHS 12 & 17 & 17 \\
\hline GAHS $<9$ & 87 & 79 \\
\hline GAHS $\geqslant 9$ & 46 & 40 \\
\hline \multicolumn{3}{|l|}{ Day 6-9 score } \\
\hline GAHS 5 & 100 & 100 \\
\hline GAHS 6 & 92 & 86 \\
\hline GAHS 7 & 94 & 87 \\
\hline GAHS 8 & 88 & 76 \\
\hline GAHS 9 & 70 & 58 \\
\hline GAHS 10 & 29 & 20 \\
\hline GAHS 11 & 39 & 28 \\
\hline GAHS 12 & 0 & 0 \\
\hline GAHS $<9$ & 93 & 86 \\
\hline GAHS $\geqslant 9$ & 47 & 37 \\
\hline
\end{tabular}

There were 134 patients with a calculable mDF. On comparison with the $\mathrm{mDF}$, the GAHS at days 1 and 7 was significantly more accurate in predicting 28 day outcome than the $\operatorname{mDF}(p=0.0016$ and $p=0.0038$ respectively). The day 1 and 7 GAHS was more accurate than the mDF score in predicting day 84 outcome $(p=0.0179$ and $p=0.0477$, respectively) (table 7 ). There were 46 patients whose coagulation was measured as an INR and had a calculable MELD score, but whose mDF was not able to be calculated. In these patients, GAHS was calculated using the INR rather than the prothrombin time ratio. On comparison with the MELD score, the GAHS day 7 was significantly more accurate in predicting 28 day outcome than the MELD score $(p=0.0339)$, but the day 1 GAHS just failed to be significantly more accurate than the MELD $(p=0.0686)$. The day 1 and 7 GAHS was more accurate than the MELD score in predicting day 84 outcome $(p=0.0005$ for both scores) (table 8 ). There were 63 patients with liver biopsy confirming alcoholic hepatitis. There were no differences in the accuracy of the GAHS between biopsied patients and 70 patients who did not have a liver biopsy (table 9).

\section{DISCUSSION}

Early identification of patients with alcoholic hepatitis at greatest risk of death is necessary for potentially beneficial treatments to be instituted. A significant proportion of patients will deteriorate after initial presentation. It would be advantageous to accurately identify patients who might benefit from intervention before their clinical condition worsened.

The variables we identified which were associated with poor outcome were similar to those noted by other observers. One other study noted that prothrombin time, white cell count, patient's age, and creatinine were related to mortality on logistic regression analysis. ${ }^{6}$ We excluded encephalopathy as a factor in our score as assessment of this can be extremely subjective in its milder forms. In addition, we did not include the presence of ascites as patients may undergo paracentesis during their admission. Thus the significance of this variable may be lost prospectively. For similar reasons, serum albumin was not used as intravenous albumin infusions are given in the management of large volume paracentesis, spontaneous bacterial peritonitis, or hepatorenal syndrome. Despite serum creatinine being a significant variable, we decided to use blood urea as a marker of renal function. This was largely to widen the clinical applicability of the score. Measurement of serum creatinine is based on the Jaffe reaction by many analysers. Unless a correction is performed, creatinine will be underestimated in the context of hyperbilirubinaemia. ${ }^{7}$ Such biochemical correction may not be immediately available, thus rendering a creatinine based score inapplicable in many clinical situations. In our initial dataset, the GAHS did perform equally well when serum creatinine, with a threshold of $100 \mu \mathrm{mol} / \mathrm{l}$, was used in place of blood urea. For similar reasons of universal applicability, we decided to use the prothrombin time expressed as a ratio of the control value. This avoids the difficulties of differing assays for prothrombin time being used in different laboratories. ${ }^{8}$

In our initial population, $\mathrm{mDF}$ was highly sensitive in the prediction of death from alcoholic hepatitis but lacked specificity. It incorrectly predicted the outcome at 28 days after admission in $51 \%$ of cases. Other practical difficulties beset the mDF. The formula relies on the absolute value of prothrombin time and, as mentioned above, there exists significant variation in the absolute values of prothrombin time obtained using different assays. ${ }^{8}$ This creates an inherent inaccuracy in the $\mathrm{mDF}$ value and limits its translation between different clinical practices.

The validation study confirmed the accuracy of the GAHS relative to the $\mathrm{mDF}$. There were however differences in the sensitivities and specificities of the GAHS between the derivation and validation datasets. GAHS was more sensitive in the validation dataset without significant loss of specificity. This may be because a large number of patients were identified as part of randomised controlled trials in two of the validation centres (Newcastle and London) whose entry criteria required an $\mathrm{mDF} \geqslant 32$. Thus patients with more severe alcoholic hepatitis would be selected, as reflected in the higher mortality in the validation population relative to

Table 7 Sensitivities (Sen), specificities (Spec), positive predictive values (PPV), negative predictive values (NPV), and overall accuracies (Acc) of the Glasgow alcoholic hepatitis score (GAHS), using the validation dataset, relative to the modified discriminant function (mDF)

\begin{tabular}{cll}
\hline & $\begin{array}{l}\text { Day 28 outcome }(\%) \\
\text { (Sen/Spec; PPV/NPV; Acc) }\end{array}$ & $\begin{array}{l}\text { Day 84 outcome }(\%) \\
\text { (Sen/Spec; PPV/NPV; Acc) }\end{array}$ \\
\hline Day 1 score & & \\
GAHS $</ \geqslant 9$ & $81 / 61 ; 47 / 89 ; 67$ & $78 / 66 ; 61 / 81 ; 71$ \\
mDF $</ \geqslant 32$ & $96 / 27 ; 36 / 93 ; 48$ & $95 / 31 ; 48 / 90 ; 57$ \\
Day 7 score & $93 / 68 ; 51 / 97 ; 75$ & $82 / 71 ; 60 / 88 ; 75$ \\
GAHS $</ \geqslant 9$ & $90 / 45 ; 36 / 93 ; 56$ & $88 / 48 ; 88 / 62 ; 62$ \\
mDF $</ \geqslant 32$ & & \\
\hline
\end{tabular}


Table 8 Sensitivities (Sen), specificities (Spec), positive predictive values (PPV), negative predictive values (NPV), and overall accuracies (Acc) of the Glasgow alcoholic hepatitis score (GAHS), using the validation dataset, relative to the MELD score

\begin{tabular}{cll}
\hline & $\begin{array}{l}\text { Day 28 outcome (\%) } \\
\text { (Sen/Spec; PPV/NPV; Acc) }\end{array}$ & $\begin{array}{l}\text { Day 84 outcome (\%) } \\
\text { (Sen/Spec; PPV/NPV; Acc) }\end{array}$ \\
\hline Day 1 score & & \\
GAHS $</ \geqslant 9$ & $75 / 68 ; 45 / 88 ; 70$ & $69 / 67 ; 45 / 85 ; 67$ \\
MELD $</ \geqslant 11$ & $92 / 29 ; 31 / 91 ; 46$ & $92 / 29 ; 31 / 91 ; 46$ \\
Day 7 score & & \\
GAHS $<1 \geqslant 9$ & $86 / 83 ; 54 / 96 ; 83$ & $86 / 83 ; 54 / 96 ; 83$ \\
MELD $</ \geqslant 11$ & $100 / 28 ; 23 / 100 ; 41$ & $100 / 28 ; 23 / 100 ; 41$ \\
\hline
\end{tabular}

Table 9 Sensitivities (Sen), specificities (Spec), positive predictive values (PPV), negative predictive values (NPV), and overall accuracies (Acc) of the Glasgow alcoholic hepatitis score (GAHS), using the validation dataset. Accuracy of the GAHS relative to biopsy proven or clinically diagnosed alcoholic hepatitis

\begin{tabular}{lll}
\hline GAHS $</ \geqslant 9$ & $\begin{array}{l}\text { Day } 28 \text { outcome (\%) } \\
\text { (Sen/Spec; PPV/NPV; Acc) }\end{array}$ & $\begin{array}{l}\text { Day } 84 \text { outcome (\%) } \\
\text { (Sen/Spec; PPV/NPV; Acc) }\end{array}$ \\
\hline $\begin{array}{l}\text { Day 1 score } \\
\text { Biopsy proven }\end{array}$ & $88 / 49 ; 55 / 86 ; 65$ & $88 / 55 ; 67 / 81 ; 71$ \\
$\quad \begin{array}{l}\text { No Biopsy } \\
\text { Day } 7 \text { score }\end{array}$ & $72 / 69 ; 41 / 89 ; 70$ & $65 / 70 ; 43 / 97 ; 69$ \\
Biopsy proven & $94 / 66 ; 63 / 95 ; 77$ & $90 / 67 ; 67 / 81 ; 77$ \\
No Biopsy & $89 / 74 ; 43 / 97 ; 77$ & $84 / 77 ; 54 / 94 ; 79$ \\
\hline
\end{tabular}

the derivation group. As the GAHS is more specific for mortality, sensitivity would also increase in a population with an overall worse prognosis.

The validation population was heterogeneous; it included patients assessed immediately on admission as well as those referred on for tertiary care. Some patients had biopsy proven alcoholic hepatitis and some had their coagulation measured using the INR rather than prothrombin time. In each of these subgroups, the score retained its accuracy which we believe supports the universal applicability of the GAHS.

Patients from whom we derived the GAHS did not have biopsy proven alcoholic hepatitis. Therefore, strictly speaking, the score assessed patients with alcoholic liver disease presenting with jaundice. However, similar clinical definitions of alcoholic hepatitis were used in the development and testing of the discriminant functions and also have been used for recruitment of patients into clinical trials. ${ }^{9-12}$ While some trials have required histological confirmation of alcoholic hepatitis, ${ }^{13}$ others have indicated that liver biopsies of those presenting with the clinical syndrome alcoholic hepatitis confirm the diagnosis in over $80 \%$ of cases. ${ }^{14}$ To further vindicate the applicability of the GAHS, the validation study indicated no difference in accuracy between biopsy proven and alcoholic hepatitis diagnosed on clinical grounds. Thus the GAHS appears to be applicable to clinical practice.

In the initial analysis, we were not in a position to calculate the MELD score as the INR was not measured in these patients, and INR and prothrombin time ratio are not necessarily equivalent. However, in the validation study it proved possible to compare the accuracy of the GAHS (using the INR rather than prothrombin time ratio) with the MELD score. The GAHS proved more accurate than the MELD score. In common with the MDF, the MELD score is similarly difficult to calculate at the bedside and inclusion of creatinine in the MELD may also limit its usefulness, as already discussed. In addition, the study by Sheth and colleagues ${ }^{5}$ did not demonstrate the superiority of the MELD score over the mDF. Another study of 98 patients with alcoholic hepatitis indicated that the MELD score was as useful as the ChildPugh score but no comparison with the mDF was made. ${ }^{15}$

In addition to informing individual patient management, an accurate scoring system would also help in structuring clinical trials in alcoholic hepatitis. On the basis of expected response from clinical studies, no published clinical study has been adequately powered to identify a significant fall in mortality with corticosteroid treatment. It might be that much of the longstanding debate regarding the use of corticosteroids is due in part to the entry criterion of an $\mathrm{mDF} \geqslant 32$ being inadequately specific for mortality.

In conclusion, we have identified factors related to mortality in patients presenting with clinical alcoholic hepatitis. Using these factors we have created a scoring system for patients which is simple to calculate and has a high degree of accuracy in predicting mortality. This score has been validated in a second data set.

\section{Authors' affiliations}

E H Forrest, Department of Gastroenterology, Glasgow Royal Infirmary, Glasgow, UK, and Victoria Infirmary, Glasgow, UK

C D J Evans, A J Morris, Department of Gastroenterology, Glasgow Royal Infirmary, Glasgow, UK

S Stewart, C P Day, University of Newcastle, Newcastle upon Tyne, UK M Phillips, J O'Grady, King's College Hospital, London, UK

Y H Oo, G Haydon, University Hospital, Birmingham, UK

P C Hayes, N C McAvoy, Department of Hepatology, Royal Infirmary of Edinburgh, Edinburgh, UK

N C Fisher, Dudley Hospitals, West Midlands, UK

S Singhal, Sandwell General Hospital, West Bromwich, UK

A Brind, University Hospital, North Staffordshire, UK

L S Murray, Division of Cardiovascular and Medical Sciences, Western Infirmary Glasgow, Glasgow, UK

Conflict of interest: None declared.

\section{REFERENCES}

1 Maddrey WC, Boitnott JK, Bedine MS, et al. Corticosteroid therapy of alcoholic hepatitis. Gastroenterology 1978;75:193-9. 
2 Carithers JRL, Herlong HF, Diehl AM, et al. Methylprednisolone therapy in patients with severe alcoholic hepatitis: a randomized multicenter trial. Ann Intern Med 1989;1 10:685-90.

3 Mathurin P, Mendenhall CL, Carithers J, et al. Corticosteroids improve shortterm survival in patients with severe alcoholic hepatitis $(\mathrm{AH})$ : individual data analysis of the last three randomized placebo controlled double blind trials of corticosteroids in severe AH. J Hepatol 2002;36:480-7.

4 McCullough AJ, O'Connor JFB. Alcoholic liver disease: proposed recommendations for the American College of Gastroenterology. Am J Gastroenterol 1998:93:2022-36.

5 Sheth M, Riggs M, Patel T. Utility of the Mayo end-stage liver disease (MELD) score in assessing prognosis of patients with alcoholic hepatitis. BMC Gastroenterol 2002;2:2.

6 Kumashiro R, Sata M, Ishii K, et al. Prognostic factors for short-term survival in alcoholic hepatitis in Japan: analysis by logistic regression. Alcoholism. Clin Exp Res 1996;20(suppl 9):383-6A

7 Lolekha PH. Sritong N. Comparison of techniques for minimizing interference of bilirubin on serum creatinine determined by the kinetic Jaffe reaction. J Clin Lab Anal 1994:8:391-9.

8 Newsome PN, Henderson NC, Germain L, et al. Prothrombin time to assess fulminant hepatic failure. Lancet $2001 ; 358: 2172$.
9 Depew W, Boyer T, Omata M, et al. Double-blind controlled trial of prednisolone therapy in patients with severe acute alcoholic hepatitis and spontaneous encephalopathy. Gastroenterology 1980;78:524-9.

10 Theodossi A, Eddleston ALWF, Williams R. Controlled trial of methylprednisolone therapy in severe alcoholic hepatitis. Gut 1982;23:75-9.

11 Mendenhall CL, Anderson S, Garcia-Pont P, et al. Short-term and long-term survival in patients with alcoholic hepatitis treated with oxdandrolone and prednisolone. N Engl J Med 1984;31 1:1464-70.

12 Akriviadis E, Botla R, Briggs W, et al. Pentoxifylline improves short term survival in severe alcoholic hepatitis: a double blind placebo controlled trial. Gastroenterology 2000;1 19:1637-48.

13 Ramond MJ, Poynard T, Rueff B, et al. A randomized trial of prednisolone in patients with severe alcoholic hepatitis. N Engl J Med 1992;326:507-12.

14 Castera L, Hartmann DJ, Chapel F, et al. Serum laminin and type IV collagen are accurate markers of histologically severe alcoholic hepatitis in patients with cirrhosis. J Hepatol 2000;32:412-8.

15 Said A, Williams J, Holden J, et al. Model for end stage liver disease score predicts mortality across a broad spectrum of liver disease. J Hepatol 2004;40:897-903

\section{Clinical Evidence - Call for contributors}

Clinical Evidence is a regularly updated evidence-based journal available worldwide both as a paper version and on the internet. Clinical Evidence needs to recruit a number of new contributors. Contributors are healthcare professionals or epidemiologists with experience in evidence-based medicine and the ability to write in a concise and structured way.

Areas for which we are currently seeking authors:

- Child health: nocturnal enuresis

- Eye disorders: bacterial conjunctivitis

- Male health: prostate cancer (metastatic)

- Women's health: pre-menstrual syndrome; pyelonephritis in non-pregnant women

However, we are always looking for others, so do not let this list discourage you.

Being a contributor involves:

- Selecting from a validated, screened search (performed by in-house Information Specialists) epidemiologically sound studies for inclusion.

- Documenting your decisions about which studies to include on an inclusion and exclusion form, which we keep on file.

- Writing the text to a highly structured template (about 1500-3000 words), using evidence from the final studies chosen, within 8-10 weeks of receiving the literature search.

- Working with Clinical Evidence editors to ensure that the final text meets epidemiological and style standards.

- Updating the text every six months using any new, sound evidence that becomes available. The Clinical Evidence in-house team will conduct the searches for contributors; your task is simply to filter out high quality studies and incorporate them in the existing text.

- To expand the topic to include a new question about once every 12-18 months.

If you would like to become a contributor for Clinical Evidence or require more information about what this involves please send your contact details and a copy of your CV, clearly stating the clinical area you are interested in, to Klara Brunnhuber (kbrunnhuber@ bmigroup.com).

\section{Call for peer reviewers}

Clinical Evidence also needs to recruit a number of new peer reviewers specifically with an interest in the clinical areas stated above, and also others related to general practice. Peer reviewers are healthcare professionals or epidemiologists with experience in evidence-based medicine. As a peer reviewer you would be asked for your views on the clinical relevance, validity, and accessibility of specific topics within the journal, and their usefulness to the intended audience (international generalists and healthcare professionals, possibly with limited statistical knowledge). Topics are usually 1500-3000 words in length and we would ask you to review between 2-5 topics per year. The peer review process takes place throughout the year, and our turnaround time for each review is ideally 10-14 days.

If you are interested in becoming a peer reviewer for Clinical Evidence, please complete the peer review questionnaire at www.clinicalevidence.com or contact Klara Brunnhuber (kbrunnhuber@bmigroup.com). 